\title{
Quantum Physics and Classical Physics - in the Light of Quantum Logic
}

\author{
Peter Mittelstaedt, University of Cologne, Germany
}

October 25, 2018

\begin{abstract}
In contrast to the Copenhagen interpretation we consider quantum mechanics as universally valid and query whether classical physics is really intuitive and plausible. - We discuss these problems within the quantum logic approach to quantum mechanics where the classical ontology is relaxed by reducing metaphysical hypotheses. On the basis of this weak ontology a formal logic of quantum physics can be established which is given by an orthomodular lattice. By means of the Solèr condition and Piron's result one obtains the classical Hilbert spaces. - However, this approach is not fully convincing. There is no plausible justification of Solèr's law and the quantum ontology is partly too weak and partly too strong. We propose to replace this ontology by an ontology of unsharp properties and conclude that quantum mechanics is more intuitive than classical mechanics and that classical mechanics is not the macroscopic limit of quantum mechanics.
\end{abstract}

\section{The dualism of Copenhagen interpretation}

Even today, 75 years after the discovery of quantum mechanics many quantum physicists are convinced that the Copenhagen interpretation is still the right way for understanding quantum physics. According to this interpretation we have to distinguish two distinct worlds, the quantum world of microscopic entities and the classical world of our everyday experience which is subject to classical physics. In the quantum world we are confronted with many strange features, complementarity, nonindividuality, nonlocality, and the loss of determinism. However, the apparatuses which measure and register the properties of the quantum system as well as the human observer, who reads the observed data are parts of the classical world that is free from the quantum physical absurdities mentioned. For describing and interpreting quantum physics we can use common language and classical logic.

We will query this doctrine here for several reasons. Firstly, during the last decades it became obvious that quantum mechanics is not restricted to the microscopic world of nuclei, atoms, and molecules but can be applied also to 
macroscopic systems. The discovery of macroscopic quantum effects like superconductivity, superfluidity, macroscopic tunnelling, etc. are strong indications that quantum physics holds also in the macroscopic world. Moreover, the successful attempts to a quantum cosmology show that quantum mechanics can even be applied on the cosmological level, to the problem of the creation of the universe and to the universe as a quantum mechanical object. Hence it seems, that there are no serious doubts today that quantum mechanics is universally valid and can be applied to all objects from elementary particles to the entire universe.

The second reason is presumably even more important. In the Copenhagen interpretation quantum mechanics is considered to be less intuitive than classical mechanics and sometimes paradoxical, whereas classical mechanics is assumed to correspond to plausible reasoning and to intuitive results. This is, however, not entirely correct. What we call "intuitive" and in accordance with "plausible reasoning" corresponds to our everyday experience, to our pre-scientific experience in the macroscopic world. However, classical physics and in particular classical mechanics is not exactly the theory of this pre-scientific experience. Classical mechanics is loaded with many hypotheses which can be traced back to the metaphysics of the $17^{\text {th }}$ and $18^{\text {th }}$ century. These metaphysical hypotheses are without any empirical counterpart, they exceed clearly our everyday experience. As examples we mention here the existence of an absolute time, the complete determination of objects, the strict causality law, and the law of conservation of substance. It is obvious that the consequences of these hypotheses are not per se intuitive in the above mentioned sense.

In quantum mechanics we are confronted with a quite different situation. Quantum mechanics may be understood as a theory of the physical reality which is free from some of the metaphysical hypotheses mentioned, i.e. quantum mechanics dispenses with some metaphysical exaggerations of the classical theory. It is important to note that quantum mechanics can be obtained from classical mechanics merely by reducing the ontological premises without incorporating new empirical components. This will be demonstrated in detail within the framework of the quantum logic approach to quantum mechanics. Consequently, in quantum mechanics just those parts of classical mechanics are missing which are not intuitive and which do not correspond to plausible reasoning. This means that quantum mechanics is more intuitive than classical mechanics - a result which is paradoxical at first glance. It is obvious that

this result together with the universal validity of quantum mechanics strongly invalidates the dualistic approach of the Copenhagen interpretation.

\section{Aiming a new quantum ontology}

The ontology of a certain domain of physics contains the most general features of the external reality which is treated in the physical domain in question. In particular the ontology should contain the material preconditions for a pragmatics which allows for the constitution of a scientific language and thus for the 
formulation of physical experience. The ontology which is underlying classical mechanics will be called classical ontology and denoted by $O(C)$. We will briefly characterise this classical ontology.

According to $O(C)$ there are individual and distinguishable objects $S_{i}$ and these objects possess elementary properties $P_{\lambda}$ in the following sense. An elementary property $P_{\lambda}$ refers to a classical object system such that either $P_{\lambda}$ or the counter property $\bar{P}_{\lambda}$ pertains to the system. An elementary property $P_{\lambda}$ can always be tested by measurement with the result that either $P_{\lambda}$ or the counter property $\bar{P}_{\lambda}$ pertains to the object. Furthermore, objects are subject to the law of "complete determination" according to which "if all possible predicates are taken together with their contradictory opposites then one of each pair of contradictory opposites must belong to it" [Kant, 1920]. Hence an object $S$ possesses each elementary property $P$ either positive $(P)$ or negative $(\bar{P})$. It follows from these strong requirements that objects can be individualised by elementary properties if impenetrability is assumed as an additional condition. For objects of the external objective reality the causality law and the law of conservation of substance hold without any restriction. Since there exist an absolute and universal time which refers to all objects of the external reality, the temporal development of these objects and their time dependent properties are strictly determined by a causal law of nature which fulfils also the conservation of substance.

There are important objections against this classical ontology. Since the metaphysical and theological reasons of Newton are no longer relevant for a justification of the ontology we have to search for alternative reasons. Are the ontological assumptions intuitive and plausible in the sense mentioned above? This is obviously not the case. The strict postulates of the classical ontology are almost in accordance with our everyday experience, but the rigorousness of the assumptions mentioned exceeds obviously the more qualitative and less rigorous prescientific everyday experience. The strict causality law, the unrestricted conservation of substance and the existence of one universal time are beyond our daily experience. These and other hypotheses of the classical ontology must not be considered as intuitive and plausible.

The second argument refers to the experimental evidence of the mentioned hypothesis. There is no experimental indication that objects can always be individualised and reidentified at later times, simply since experiments which would confirm this assumption have never been performed in classical physics. In addition, the principle of complete determination mentioned above has never been tested with an accuracy which would allow to call the result a principle. Consequently, there is no justification for a strict causality law such that the present state of an object allows for predictions about all elementary properties. Hence we find that there is no empirical justification for the classical ontology $O(C)$. Instead, the classical ontology is based on hypotheses whose origin can be traced back to the metaphysics of the $17^{\text {th }}$ and $18^{\text {th }}$ century.

The classical ontology is neither intuitive and plausible nor is it justified by experimental evidence. Moreover, - what is more important - the classical ontology $O(C)$ is not in accordance with quantum physics. A quantum me- 
chanical object system does not possess all possible elementary properties $P_{\lambda}$ either positive $\left(P_{\lambda}\right)$ or negative $\left(\bar{P}_{\lambda}\right)$. It is not carrier of all possible properties. Instead, only a subset of all properties pertains to the system and can simultaneously be determined. These properties are often called "objective" properties and they pertain to the object like in classical ontology. From these restrictions it follows that in quantum mechanics no strict causality law can be established and that object systems cannot be individualised and reidentified by means of their objective properties.

We will not use here these empirical results for a reconstruction of an ontology for quantum phenomena. However, we learn from these considerations, that the classical ontology is not only based on classical metaphysics and partially hypothetical, but that classical ontology contains too much structure and too strong requirements compared with quantum physics. This observation offers the interesting possibility to formulate the ontology $O(Q)$ of quantum physics by relaxing and weakening some hypothetical requirements of the classical ontology $O(C)$. It is important to note that no new requirements must be added to the assumptions of the classical ontology. Quantum ontology can thus be formulated as a reduced version of the classical ontology $O(C)$ :

$O(Q)$-1: If an elementary property $P$ pertains to a system as an objective property, then a test of this property by measurement will lead with certainty to the result $P$. In addition, any arbitrary elementary property $P$ can be tested at a given object with the result that either $P$ or the counter property $\bar{P}$ pertains to the object system. (These requirements are in complete accordance with $O(C)$ ).

$O(Q)-2$ : Quantum objects are not completely determined. They possess only a few elementary properties either positive or negative. Properties which pertain simultaneously to an object are called "objective" and "mutually commensurable".

$O(Q)$-3: For quantum objects there is no strict causality law, simply since the present state of an object system is never completely determined.

$O(Q)-4$ : The lack of complete determination and of strict causality implies that quantum objects cannot be individualised and reidentified at later times.

The mutual relations between classical and quantum ontology are the key for the very intertheoretical relations between classical and quantum physics. This will become obvious in the quantum logic approach to quantum mechanics when quantum ontology is used as starting point for establishing a formal language and logic of quantum physics. This way of reasoning will be made explicit in the following section. 


\section{The quantum logic approach to quantum me- chanics}

\subsection{Language, semantics, and pragmatics}

On the basis of the quantum ontology $O(Q)$ described above we will establish a formal language of quantum physics. Let $S$ be a proper quantum system and $A, B, \ldots$ elementary propositions which attribute predicates (properties) $P(A), P(B), \ldots$ to system $S$ at times $t_{1}, t_{2}, \ldots$ Hence, we write for elementary propositions $A\left(S, t_{1}\right), B\left(S, t_{2}\right), \ldots$ According to $O(Q)$-1 we assume that for every elementary proposition $A$ there exists a finite testing procedure which shows whether $P(A)$ pertains to $S$ or not. If $P(A)$ pertains to $S$ at time $t_{1}$, then the proposition $A\left(S, t_{1}\right)$ is called to be "true", otherwise $A\left(S, t_{1}\right)$ is said to be "false". The assumption, that for every elementary proposition there is a testing procedure which decides between "true" and "false" means, that these propositions are "value definite". Hence, an elementary proposition can either be proved (with result $A$ ) or disproved (with result $\bar{A}$ ), where $\bar{A}$ is the counter proposition of $A$. Furthermore, we assume that after a successful proof of $A$ new proof attempt leads with certainty to the same result, provided the time interval between the two proof attempts is sufficiently small. This requirement is again in accordance with $O(Q)-1$. Since after the first test the property $P(A)$ pertains objectively to the system and can thus be tested with the certain result $P(A)$. This assumption means that there are repeatable measurement processes, which can be applied to the testing procedures. However, - and this is an important restriction of $O(C)$ - if after a successful proof of $A$, say, another proposition $B$ is proved, then a new proof attempt of $A$ will in general not lead to the previous result. Hence, we will not assume that two propositions $A$ and $B$ are in general simultaneously decidable. If accidentally two propositions $A$ and $B$ are always jointly decidable, we will call $A$ and $B$ "commensurable". In this case, after the proof attempt of $B$ the result of the previous $A$-test is still available. However, in the general case the result of a previous test is only restrictedly available.

On the basis of the set $\mathcal{S}_{Q}$ e of elementary propositions we introduce the logical connectives by the possibilities to attack or to defence them, i.e. by the possibilities to prove or to disprove the connective. Here, we consider the sequential conjunction $A \sqcap B(A$ and then $B)$ which refers to two subsequent instants of time $t_{1}$ and $t_{2}$ with $t_{1}<t_{2}$ and the logical connectives $\neg A$ (not $\left.\mathrm{A}\right)$, $A \wedge B(\mathrm{~A}$ and $\mathrm{B}), A \vee B(A$ or $B)$, and $A \rightarrow B$ (if $A$ then $B)$ - which refer to one simultaneous instant of time. The definitions of the sequential and logical connectives by attack-and defence schemes can be illustrated most conveniently by chronologically ordered proof trees. Correspondingly, in the proof tree of the sequential conjunction $A \sqcap B$, the first branching point corresponds to a $A$-test at $t_{1}$, the second one to a $B$-test at $t_{2}$. Note, that for the truth of $A \sqcap B$ the commensurability of $A$ and $B$ does not matter. However, for the proof trees of the logical connectives, which refer to one simultaneous instant of time, the commensurabilities of the elementary propositions play an important role. 
The concepts of truth and falsity of a compound proposition which is composed by the connectives can then be defined by success and failure in a proof tree, respectively. [Mittelstaedt, 1978; Stachow, 1980; Mittelstaedt, 1987].

Furthermore, we will define here binary relations between propositions. First, the proof equivalence $A \equiv B$ means that $A$ can be replaced in any proof tree of a compound proposition by $B$ without thereby changing the result of the proof tree. Second, the value equivalence $A=B$ means that $A$ is true (in the sense of a proof tree) if and only if $B$ is true. Third, the relation of implication $A \leq B$ can be defined by $A \equiv A \wedge B$. Hence, the two implications $A \leq B$ and $B \leq A$ imply the proof equivalence $A \equiv B$. Finally, we mention that $A \rightarrow B$ is true if and only if $A \leq B$ holds. The full quantum language $\mathcal{S}_{Q}$ can then inductively be defined by the set $\mathcal{S}_{Q}{ }^{\mathrm{e}}$ of elementary propositions and the connectives mentioned. Together with the always true elementary proposition $\mathrm{V}$, the always false elementary proposition $\Lambda$, and the three relations one obtains the language $\mathcal{S}_{Q}$.

\subsection{Quantum logic}

The semantics described here is a combination of a realistic semantics (for elementary propositions) and a proof semantics (for connectives). Hence, the truth of a compound proposition depends on the connectives contained in it as well as on the elementary propositions and their truth values. However, there are finitely connected propositions which are true in the sense of the semantics mentioned, irrespective of the truth values of the elementary propositions contained in it. These propositions are called formally true. - The precondition that measurements are repeatable implies that $A \rightarrow A$, the law of identity, is formally true. The value definiteness of elementary propositions implies that also finitely connected propositions are value definite and thus $A \vee \neg A$, the tertium non datur law, is formally true. In a similar way, it follows that $\neg(A \wedge \neg A)$, the law of contradiction, and $(A \wedge(A \rightarrow B)) \rightarrow B$, the modus ponens law, are formally true. - Formally true propositions can also be expressed by "formally true implications". E.g. the modus ponens law reads $A \wedge(A \rightarrow B) \leq B$. In addition, the relations $A \leq \mathrm{V}$ and $\Lambda \leq A$ hold for all propositions $A \in \mathcal{S}_{Q}$. The formal truth of a proposition $A$ can then be expressed by $\mathrm{V} \leq A$. E.g. the tertium non datur law reads $\mathrm{V} \leq A \vee \neg A$.

There are two kinds of propositions $A \in \mathcal{S}_{Q}$. If a compound proposition contains in addition to elementary propositions only the logical connectives $\wedge, \vee, \neg$ and $\rightarrow$, then it is called a "logical proposition". In the more general case, when the proposition contains also sequential connectives, in particular the sequential conjunction $\square$, then it is called a "sequential proposition". In addition to the formally true logical propositions mentioned above, there are also formally true sequential propositions. If $A$ and $B$ are logical propositions then $A \wedge B \leq A \sqcap B$ is a formally true implication. The totality of formally true implications can be summarised in a calculus which contains "beginnings" $\Rightarrow A \leq B$ and rules $A \leq B \Rightarrow C \leq D$. Here, we distinguish the calculus $\mathbf{L}_{Q}$ of formally true logical propositions and the calculus $\mathbf{S}_{Q}$ of formally true sequential 
propositions. [Mittelstaedt, 1978; Stachow, 1980].

For an algebraic characterisation of the calculi $\mathbf{L}_{Q}$ and $\mathbf{S}_{Q}$ we consider the corresponding Lindenbaum-Tarski algebras. The Lindenbaum-Tarski algebra of the calculus $\mathbf{L}_{Q}$ is given by a complete, orthomodular lattice $L_{Q}$. Subsets of mutually commensurable propositions constitute a Boolean sublattice $L_{B} \subseteq L_{Q}$ of the lattice $L_{Q}$ [Mittelstaedt, 1987]. Moreover, if the entire quantum language $\mathcal{S}_{Q}$ refers to one individual quantum system, then the lattice $L_{Q}$ is atomic and fulfils the covering law [Stachow, 1984]. In this case we denote the lattice by $L_{Q}^{*}$. The Hilbert lattice $L_{H}$ of projection operators in Hilbert space can be obtained from the lattice $L_{Q}^{*}$ by adding the Solér law, the meaning of which is, however, still open [Solér, 1995]. Correspondingly, the Lindenbaum-Tarski algebra of the calculus $\mathbf{S}_{Q}$ of sequential quantum logic is given by a Baer* semigroup. [Stachow, 1980; Foulis,1960]. It is well known that by means of a result by Piron [Piron, 1976] from the lattice $L_{H}$ the three classical Hilbert spaces can be obtained and that for the complex numbers $\mathbb{C}$ quantum mechanics in Hilbert space is achieved.

\subsection{Is quantum mechanics a priori valid?}

The described approach to quantum mechanics which starts from the relaxed quantum ontology $O(Q)$ and leads finally to the quantum mechanical Hilbert space, is sometimes considered as an a priori justification of quantum theory [Mittelstaedt, 1978]. The term "a priori" seems to be legitimated here, since the starting point of this approach are the most general preconditions of a scientific language of physics, i.e. the assumptions of the weak ontology $O(Q)$. However, this way of reasoning is not fully convincing. Firstly, up to now there is no plausible and intuitiv justification of Solér's law, which appears in the present approach as an additional ad hoc assumption. Hence, one could ask whether the quantum ontology $O(Q)$ is really the right starting point. $O(Q)$ is too weak, since the main restriction of quantum ontology with respect to classical ontology, the complementarity requirement, is a very strong postulate. Two properties which are not commensurable for accidental reasons are complementary in the sense that they cannot be tested simultaneously. Complementarity in this strong form must be required in quantum mechanics for sharp observables which are given by $\mathrm{PV}$-measures.

However, even in quantum mechanics the strong complementarity requirement can be relaxed by the uncertainty principle making use of unsharp observables in the sense of POV-measures. POV-measures are the most general observables which allow for a probability interpretation of quantum mechanics [Busch et al. 1995]. Two unsharp properties of a quantum system can be attributed jointly to the object, if the conveniently defined degrees of unsharpness of the two properties fulfil the Heisenberg uncertainty inequality [Busch, 1985]. Obviously, a quantum ontology $O\left(Q^{\mathrm{u}}\right)$ which replaces the complementarity requirement by the uncertainty principle, is somewhat stronger than the original ontology $O(Q)$.

The ontology $O(Q)$ is not only too weak but - with respect to another feature 
- also too strong. In accordance with the classical ontology $O(C)$ we assumed in $O(Q)-1$ that any elementary property $P$ can be tested experimentally with the result that either $P$ or the counter property $\bar{P}$ pertains to the system. This ontological precondition implies that elementary propositions of the quantum language are value definite and that the tertium non datur holds in quantum $\operatorname{logic} \mathbf{L}_{Q}$. However, the ontological precondition that any elementary property can be tested by experiment (with the result $P$ or $\bar{P}$ ) exceeds the possibilities of Hilbert space quantum mechanics. Within the framework of the quantum theory of unitary premeasurements it follows that pointer objectification cannot be achieved for closed systems [Mittelstaedt 1998]. Hence, value definiteness of elementary propositions is incompatible with quantum mechanics in Hilbert space and must be relaxed in some sense. In this situation it suggests itself to begin with elementary propositions that are not value definite and correspond to unsharp properties given by POV-measures ${ }^{1}$ Hence it seems that also the second objection against the quantum ontology $O(Q)$ can be taken account of by the quantum ontology $O\left(Q^{\mathrm{u}}\right)$ based on unsharp properties.

Hence, on the basis of the slightly modified quantum ontology $O\left(Q^{\mathrm{u}}\right)$ a fresh start by means of unsharp properties seems to be a quite promising attempt. In a first step of this approach a formal language and logic of not necessarily value definite quantum mechanical propositions must be developed. In a second step from the algebraic structure of the logic the algebra of effects and the Hilbert space must be reconstructed. Hence one could either start from a language of unsharp propositions or from a modified algebraic structure of quantum logic. In recent years many interesting logical systems for unsharp propositions were proposed. [Dalla Chiara 1993, 1994, 1995, Foulis 1997, Giuntini 1989, 1990, Mittelstaedt 1978]

However, it is still an open question whether in this way a consistent operational approach to quantum mechanics can be obtained. Up to now the logical systems mentioned were not yet reconstructed in an operational way starting from a formal language of unsharp propositions. Furthermore, the LindenbaumTarski algebra of a logical calculus of unsharp propositions is not per se equivalent to the algebra of effects in Hilbert space. We do not know which kind of law must be added to the algebra of unsharp propositions in order to obtain the effect algebra mentioned (it could be as complicated as the Solér law). Even the last step of an operational foundation of quantum physics, the way from the effect algebra to the Hilbert space requires more detailed investigations.

\subsection{The ontological priority of quantum mechanics}

Although the task of reconstructing quantum mechanics on the basis of a logic of unsharp propositions is not yet finally performed, we can draw already some interesting conclusions which refer to the interpretation of quantum mechanics. The basis of this approach, the (uncertainty) ontology $O\left(Q^{\mathrm{u}}\right)$ is somewhat

\footnotetext{
${ }^{1}$ It must be mentioned that up to now it is not yet quite clear whether the problem of pointer objectification can completely be solved by POV-measures [Busch 1998].
} 
richer than the (complementarity) ontology $O(Q)$ but weaker than the classical ontology $O(C)$ of complete determination. This classical ontology is not only based on experience but also on several metaphysical hypotheses - which are weakened or cancelled in $O\left(Q^{\mathrm{u}}\right)$.

Since these metaphysical hypotheses (complete determination, individuality, and full determinism) clearly exceed our everyday experience, and since we call phenomena intuitive and understandable if they are in accordance with this everyday experience, classical mechanics is not thoroughly intuitive. However, since the hypotheses contained in the classical ontology $O(C)$ are strongly reduced in the (uncertainty) ontology $O\left(Q^{\mathrm{u}}\right)$ as well as in the (complementarity) ontology $O(Q)$, we expect that the implications of the quantum ontology $O\left(Q^{\mathrm{u}}\right)$ are more intuitive and more plausible than the implications from the classical ontology.

In particular the quantum logic approach can further illustrate this result. The logical systems which follow from the ontologies $O(Q)$ and $O\left(Q^{\mathrm{u}}\right)$ are based on weaker and less hypothetical pragmatic preconditions than the Boolean lattice $L_{B}$ of classical logic. Hence the resulting quantum mechanics is more intuitive and more plausible than classical mechanics. In addition, since quantum mechanics is based on weaker premises than classical mechanics, it is nearer to the "truth" than classical mechanics.

On the basis of these results we can formulate the rôle of classical mechanics. Firstly, classical mechanics is loaded with metaphysical hypotheses which clearly exceed our everyday experience. Since quantum mechanics is based on strongly relaxed hypotheses of this kind, classical mechanics is less intuitive and less plausible than quantum mechanics. Hence classical mechanics, its language and its logic cannot be the basis of an adequate interpretation of quantum mechanics. Secondly, classical mechanics is not the limit of quantum mechanics for macroscopic phenomena. Since quantum mechanics of closed systems does not explain the objectification, i.e. the classical behaviour of pointer values, classical mechanics cannot be the macroscopic limit of quantum mechanics. However, this argument which is still subject of controversial debates, is not the main reason. The essential argument which shows that classical mechanics is not the limiting case of quantum mechanics is based on the observation that classical mechanics is loaded with metaphysical hypotheses without any empirical counterpart. Since some of these hypotheses are explicitly eliminated in quantum theory, it is obvious that there is no approximation procedure which leads from quantum mechanics to classical mechanics. Classical mechanics describes a fictitious world which does not exist in reality.

\section{References}

[1] Busch, P: Can unsharp objectification solve the measurement problem, Int. J. Theor. Phys. 37, 1998, 241-47. 
[2] Busch, P.: Indeterminacy Relations and Simultaneous Measurements in Quantum Theory, Int. J. Theor. Phys. 24, 1985, 63-92.

[3] Busch, P., P. Lahti, and P. Mittelstaedt: The Quantum Theory of Measurement, 2nd. ed.1996, Springer-Verlag, Heidelberg.

[4] Dalla Chiara, M. L.: Unsharp Quantum Logics, Int. J. Theor. Phys. 34, 1995, 1331-1336.

[5] Dalla Chiara, M.L., Cattaneo G. and Giuntini, R.: Fuzzy-intuitionistic quantum logic, Studia Logica, 52, 1993, 1-24.

[6] Dalla Chiara, M. L. and Giuntini, R.: Partial unsharp quantum logics, Found. of Physics 24, 1994, 1161-1177.

[7] Foulis, D. J.: Baer*-Semigroups, Proceedings of the American Mathematical Society, 11, 1960, 648-654.

[8] Foulis, D. J. and Bennett, M. K.: Effect algebras and unsharp quantum logics, Found. of Physics 24 1994, 1331-1352.

[9] Giuntini, R.: Brower-Zadeh logic and the operational approach to quantum mechanics, Found. of Physics 20, 1990, 701-714.

[10] Giuntini, R. and Greuling, H.: Towards a language of unsharp properties, Found. of Physics 20, 1989, 931-935, 1989.

[11] Kant, I.: Critique of Pure Reason, translated by N. K. Smith, Macmillan 1929, B600.

[12] Mittelstaedt, P.: Quantum Logic, D.Reidel Publ. Co., Dordrecht, 1978.

[13] Mittelstaedt, P.: Language and Reality in Quantum Physics, in: Symposium on the Foundations of modern Physics, 1987, World Scientific, Singapore, 1987, 229-250.

[14] Mittelstaedt, P.: The Interpretation of Quantum Mechanics and the Measurement Process, Cambridge University Press, Cambridge 1998.

[15] Piron, C.: Foundations of Quantum Physics, W.A. Benjamin, Reading, Mass. 1976.

[16] Solèr, M. P.: Characterisation of Hilbert Spaces by Orthomodular Lattices, Communications in Algebra, 23 (1), 1995, 219-243.

[17] Stachow, E. W.: Logical Foundation of Quantum Mechanics, Inter. Journ. of Theor. Phys., 19, 1980, 251-304.

[18] Stachow, E. W.: Structures of quantum language for individual systems, in: Recent Developments in Quantum Logic, P. Mittelstaedt and E.-W. Stachow, eds., BI-Wissenschaftsverlag, Mannheim, 1984, 129-145. 